\title{
Instant SNR Estimation on Digital Symbols
}

\author{
Mete Yildırım $^{1 *}$, Radosveta Sokullu ${ }^{2}$ \\ $\mathbf{1}^{*}$ Ege University, Faculty of Engineering, Departmant of Electrical and Electronics, İzmir, Turkey, (ORCID: 0000-0001-6335-4752), meteyildirim2005@gmail.com \\ ${ }^{2}$ Ege University, Faculty of Engineering, Departmant of Electrical and Electronics, İzmir, Turkey, (ORCID: 0000-0002-3544-0319), radosveta.sokullu@ege.edu.tr
}

(First received 28 December 2020 and in final form 7 November 2021)

(DOI: $10.31590 /$ ejosat.848274)

\begin{abstract}
ATIF/REFERENCE: Yıldırım, M. \& Sokullu, R. (2021). Instant SNR Estimation on Digital Symbols. European Journal of Science and Technology, (27), 644-648.

Abstract

Signal-to-noise ratio is a very important parameter that must be known in order to make many communication systems to work efficiently. The determination of Signal-to-noise ratio may cause extra cost, complexity or can cause a decrease in the efficiency of resource allocation depending on the method used. The machine learning methods offer a practical solution while eliminating these negative aspects with supervised learning and offline training. Deep learning stands out with its success as a type of machine learning. In this study, the estimation of the instantaneous value of the signal to noise ratio in digital symbols was investigated using the deep learning technique.
\end{abstract}

Keywords: Communication, Deep Learning, Estimation, Machine Learning, SNR

\section{Sayısal Sembollerde Anlık SNR Kestirimi}

Öz

Sinyal-gürültü oranı, birçok haberleşme sisteminin verimli çalışabilmesi için bilinmesi gereken çok önemli bir değerdir. Bu değerin belirlenmesi, kullanılan yönteme bağlı olarak ekstra maliyete, karmaşıklığa veya kaynak tahsisinin verimliliğinde düşüşe neden olabilir. Makine öğrenimi yöntemleri, denetimli öğrenme ve çevrimdışı eğitim ile bu olumsuz yönleri ortadan kaldırırken pratik çözüm yolları sunarlar. Derin öğrenme, bir tür makine öğrenimi olarak başarısıyla öne çıkmaktadır. Bu çalışmada, dijital sembollerdeki sinyal-gürültü oranının anlık değerinin tahminin derin öğrenme tekniği kullanılarak yapılması incelenmiştir.

Anahtar Kelimeler: Haberleşme, Derin Öğrenme, Kestirim, Makine Öğrenimi, SNR

\footnotetext{
*Corresponding Author: meteyildirim2005@gmail.com, mete.yildirim@mail.ege.edu.tr
} 


\section{Introduction}

Signal to Noise Ratio (SNR) is vitally important parameter for all telecommunication systems. The evaluation of performance of communication systems, spectrum management, relay selection in cooperative communication, channel resource allocation, adaptive modulation \& coding and transmission power control requires prior knowledge of SNR. Besides, for optimal performance, many systems require knowledge of SNR (Bogale \& Vandendorpe, 2014; Nandakumar et al., 2019; Rasouli \& Anpalagan, 2010; Santos Filho et al., 2018; Thilina, Choi, Saquib, \& Hossain, 2013; Xiang, Johnston, \& Le Goff, 2017; Xie, Zhang, Guo, \& Zhao, 2019; Yıldırım \& Ilhan, 2014).

The SNR estimation methods can be classified into two: Data aided and Non-data aided estimation (Abeida, 2010; Salman, Badawy, Elfouly, Khattab, \& Mohamed, 2014). The first one generally requires the transmission of a data that is known to receiver. This data can be in various size and has to be transmitted very often during transmission. The duration and frequency of this data may change with the channel condition. Definitely this introduces waste of resources. On the other hand, this method is very practical to use due to its simplicity. Furthermore, the data transmiited for channel estimation and equalization can also be used for SNR estimation. In this case the amount of useless data is reduced but not completely eliminated. This approche does not use some data to estimate SNR but use more complex algorithm that requires more power, calculation capacity and etc.

Machine learning, especially deep learning has proved to be very useful in many fields. It can reduce complex mathematical burden to a simple training and prediction procedure. A many time, the researchers in the field of electronic communication has to deal complex mathematical equations to introduce solutions to the existing problems. This has become more apparent with scarcity of resources and use of higher frequencies. Hence, we observe many studies that use deep learning as a tool to address the problems. Not all but among some important works, in (Farsad \& Goldsmith, 2018; Samuel, Diskin, \& Wiesel, 2019; Ye, Li, \& Juang, 2017) signal detection, in (Farsad, Rao, \& Goldsmith, 2018; Gruber, Cammerer, Hoydis, \& Brink, 2017; Nachmani et al., 2018) channel encoding and decoding, in (He, Wen, Jin, \& Li, 2018; Wang, Narasimha, \& Heath, 2018; Wen, Shih, \& Jin, 2018) channel estimation, prediction, and compression, in (Challita, Dong, \& Saad, 2018; Daniels \& Heath, 2009; Sun et al., 2018; Va, Choi, Shimizu, Bansal, \& Heath, 2017) resource allocation are studied.

Driven with influence of deep learning (DL) on addressing problem solutions related to electronics communication system, in this study, we examine the performance of instant SNR estimation with DL on binary shift key modulated symbol in additive white gaussian channel with optimized model approach.

In the next section, we introduce deep learning theory briefly.

\section{Deep Learning}

The learning of Neural network is generally categorized into two classes namely supervised and unsupervised learning. The unsupervised learning is evaluated as the most promising learning method yet it is considered in its baby state. Hence, the supervised learning is mostly used learning strategy currently. The supervised learning requires only well labelled data. This data is used to train a model. Then, the trained model is used for prediction either for classification or regression procedure. The trained model is produces nothing but a matrix that is used to multiply input the produce output. The matrix size defined with the number of input features, output classes, neurons and layers.

The deep learning model is nothing but a neural network with more and more layers, neurons and, complex connections. The deep learning model capacity has not been proved mathematically yet. Hence the studies aim to provide better results to the existing results for a particular study practically by building and training the developed models by inspiration.

The deep learning models often categorize as convolution neurol network $(\mathrm{CNN})$, recurrent neural network $(\mathrm{RNN})$ and fully connected neurol network (DNN). CNN mostly is used in image processing, $\mathrm{RNN}$ is used natural language processing and DNN is a general structure more likely ordinary NN and it may be used where $\mathrm{NN}$ is employed mostly.

The fully connected(dense) neurol network is a feedforward NN. It uses iterative processing and maps the input features to the desired output. The mapping from a layer to the next layer is given as $r_{i}=f_{i}\left(r_{i-1}, \theta_{i}\right), i=1, \ldots I$ for $I$ iteration, where $\theta_{i}=\left(W_{i}, b_{i}\right)$ is the set of parameters. In the case of dense layer, the mapping takes the form of $\sigma\left(W_{i} r_{i-1}+b_{i}\right)$, where $\sigma($.$) is$ known activation function. The objective of the training is to minimize the loss $L(\theta)=\frac{1}{S} \sum_{k=1}^{S} l\left(r_{L, k}^{*}, r_{L, k}\right)$ where $S$ is number of training data, $l($.$) is the lost for k^{\text {th }}$ training input, $L($.) is the average loss. The lost function is chosen to with respect to objective. For example, for multiclass case Categorical cross-entropy or for regression mean squared estimation (MSE) may be desired choice. The stochastic gradient decent is well known algorithm to find the set of parameters $\theta$. The SGD uses random initial values, $\theta=\theta_{0}$, which are generally very small numbers and taken from some well-known distribution (ex. Normal distrubition) and updates as $\theta_{t+1}=\theta_{t}-\eta \nabla \tilde{L}\left(\theta_{t}\right)$ where $\eta$ is the step size or learning rate, $\nabla($.$) is the gradient and$ $\widetilde{L}$ is a batch from training set. The more on the theory can be found in (Goodfellow, Bengio, \& Courville, 2016).

\section{The Model Implementation}

\subsection{Step 1: Data Preparing}

The first step of any machine learning is the prepare data intended to use to train the model. The designer should decide what data with what features are to be used. The baseband communication requires no up-conversion and the data is transmitted only after modulation. The bandpass modulation requires up-conversion but it can be represented in baseband with complex form. In summary, a received symbol may be given in the form of $a+j b$ where we consider the noise is already added and it can represent any form of amplitude-phase modulation. The noise is considered, AWGN, the independent but identically distributed for every symbol. We assume we already know the 
power of added noise in $\mathrm{dB}$. The number of features of data basically only two since the data has only two dimensions. That is a and $\mathrm{b}$. We know that the NN model cannot make use of the imaginary part, hence we only use its amplitude. Some samples data are given in Table 1. This is only for demonstration purpose. We mean that the input data can be organized in a variety of ways such as more symbols can be arranged to apply the inputs of model as long as it is ensured that all the symbols applied to input at the same time has the same power of the noise. If only one symbol is decided to apply to the model input, some feature engineering can be considered to apply. Generally, we need so many symbols to train a model. So many symbols are created then they are put in the form of Table.1. The Figure 1 shows BPSK symbols at the receiver and transmitter end. The received symbols are noisy but for clear demonstration it is kept relatively small and in realty it can be severely bad.

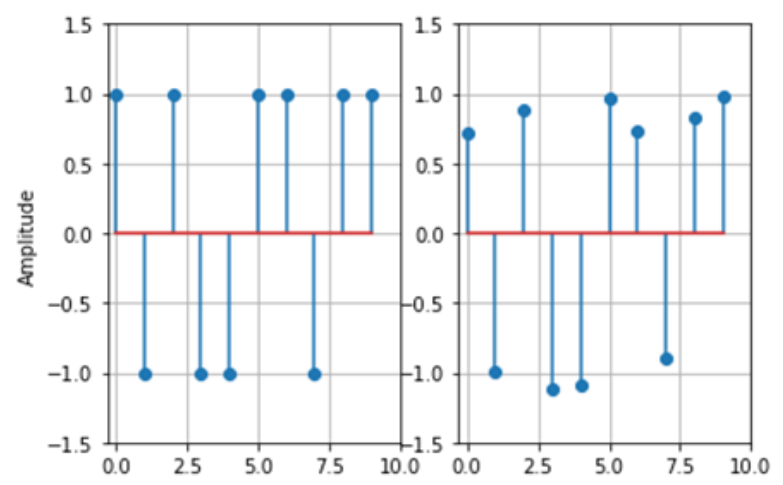

Figure 1. The transmitter and the received information

\subsection{Step 2: Training, Evaluation and Testing}

The prepared data is divided into three. We keep \%20 and $\% 10$ for evaluation and testing of the model while the rest is kept for training. The model given in Figure 4 is used and trained. As seen from the figure, 8 symbols are used for input feeding. The model uses tanh (1) for all layers except last layer which keeps the default, the linear activation function. Adam is used as optimizer for the model while mean square error (MSE) used as loss function.

$$
\tanh (x)=\frac{\exp (x)-\exp (-x)}{\exp (x)+\exp (-x)}
$$

The model learning rate is defined as 0.001 and the number of epochs is set 80 . The training and validation loss is shown Figure 2. The figure promises well-fitted behavior hence we are assured of model success.

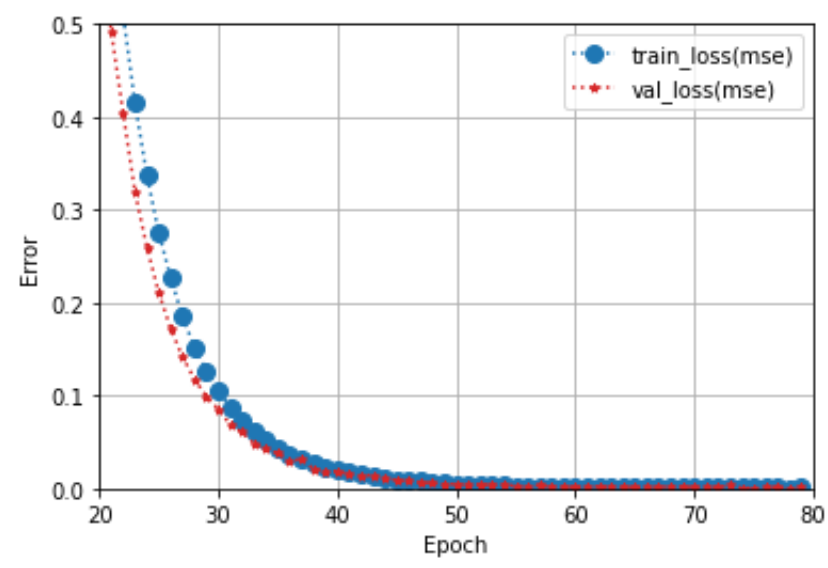

Figure 2. Model loss

The model is tested on the testing data. The results are both represented in both tabularly and graphically. The samples MSE from Table 2 shows that the average error is on the order of $10^{-2}$ . The estimated and ground truth noise level given Figure 3 shows promising matching pattern.

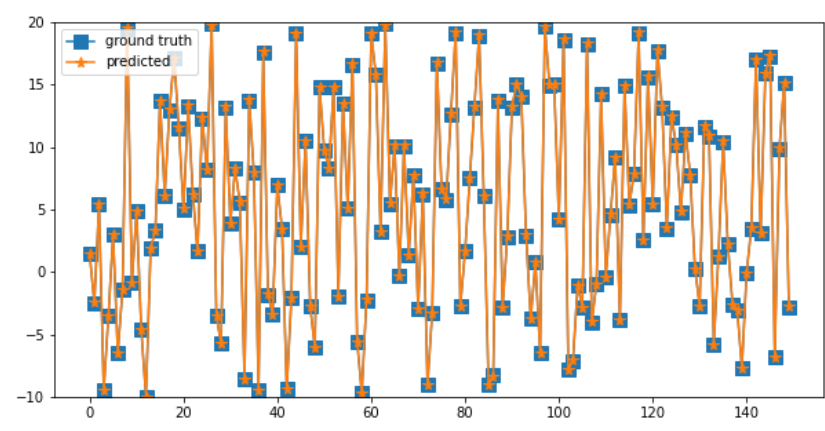

Figure 3. Real SNR $(d B)$ and predicted $S N R$

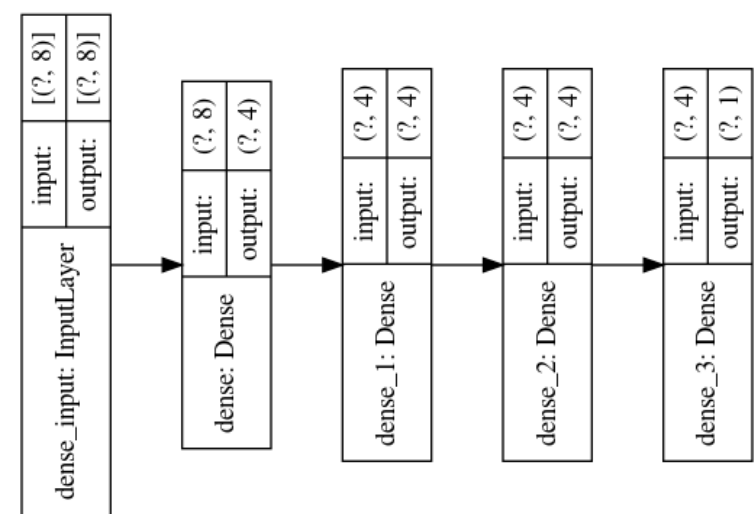

Figure 4. The fully connected model 
Table 1. The data samples

\begin{tabular}{rccc}
\hline Tx Symbol & Rx Symbol & Input Features & Ground Truth(dB) \\
\hline 1 & $-0.746287-1.746287 j$ & $(-0.746287,-1.746287)$ & 0 \\
\hline 1 & $2.97974585+1.97974585 \mathrm{j}$ & $(2.979745,1.979745)$ & -5 \\
\hline-1 & $-0.94145464+0.05854536 \mathrm{j}$ & $(-0.941454,0.058545)$ & 10 \\
\hline
\end{tabular}

Table 2. Real SNR vs. Predicted

\begin{tabular}{cc}
\hline Test Data SNR in dB & Estimated SNR in dB \\
\hline 3.558581 & 3.5815442 \\
\hline 6.887164 & 6.852639 \\
\hline 4.028512 & 4.0411315 \\
\hline-0.539802 & -0.5364828 \\
\hline 12.707294 & 12.694737 \\
\hline 6.487479 & 6.4526787 \\
\hline-6.809761 & -6.7699556 \\
\hline 4.572642 & 4.5716085 \\
\hline-5.765522 & -5.725233 \\
\hline-6.447449 & -6.4061546 \\
\hline
\end{tabular}

\section{Conclusions}

The instant SNR estimation of digital symbols are done in AWGN channel. A deep learning model is used for estimation. The model is trained offline and estimation is done instantly. This can be considered as non-data aided estimation method. The superiority of the machine learning method stems from the fact that it does not require complex calculations such as non-data aided estimation, and consequently it reduces the cost and promises more speed.

\section{References}

Abeida, H. (2010). Data-aided SNR estimation in time-variant Rayleigh fading channels. IEEE transactions on signal processing, 58(11), 5496-5507. IEEE.

Bogale, T. E., \& Vandendorpe, L. (2014). Max-Min SNR signal energy based spectrum sensing algorithms for cognitive radio networks with noise variance uncertainty. IEEE transactions on wireless communications, 13(1), 280-290. IEEE.

Challita, U., Dong, L., \& Saad, W. (2018). Proactive resource management for LTE in unlicensed spectrum: A deep learning perspective. IEEE transactions on wireless communications, 17(7), 4674-4689. IEEE.

Daniels, R. C., \& Heath, R. W. (2009). An online learning framework for link adaptation in wireless networks. 2009 Information Theory and Applications Workshop (pp. 138140). IEEE.

Farsad, N., \& Goldsmith, A. (2018). Neural network detection of data sequences in communication systems. IEEE Transactions on Signal Processing, 66(21), 5663-5678. IEEE.

Farsad, N., Rao, M., \& Goldsmith, A. (2018). Deep learning for joint source-channel coding of text. 2018 IEEE International
Conference on Acoustics, Speech and Signal Processing (ICASSP) (pp. 2326-2330). IEEE.

Goodfellow, I., Bengio, Y., \& Courville, A. (2016). Deep Learning. MIT Press.

Gruber, T., Cammerer, S., Hoydis, J., \& Brink, S. ten. (2017). On deep learning-based channel decoding. 2017 51st Annual Conference on Information Sciences and Systems (CISS) (pp. 1-6). IEEE.

He, H., Wen, C.-K., Jin, S., \& Li, G. Y. (2018). Deep learningbased channel estimation for beamspace mmWave massive MIMO systems. IEEE Wireless Communications Letters, 7(5), 852-855. IEEE.

Nachmani, E., Marciano, E., Lugosch, L., Gross, W. J., Burshtein, D., \& Be'ery, Y. (2018). Deep learning methods for improved decoding of linear codes. IEEE Journal of Selected Topics in Signal Processing, 12(1), 119-131. IEEE.

Nandakumar, S., Velmurugan, T., Thiagarajan, U., Karuppiah, M., Hassan, M. M., Alelaiwi, A., \& Islam, M. M. (2019). Efficient Spectrum management techniques for cognitive radio networks for proximity service. IEEE Access, 7, 4379543805. IEEE.

Rasouli, H., \& Anpalagan, A. (2010). SNR-based vs. BER-based power allocation for an amplify-and-forward single-relay wireless system with MRC at destination. 2010 25th Biennial Symposium on Communications (pp. 429-432). IEEE.

Salman, T., Badawy, A., Elfouly, T. M., Khattab, T., \& Mohamed, A. (2014). Non-data-aided SNR estimation for QPSK modulation in AWGN channel. 2014 IEEE 10th International Conference on Wireless and Mobile Computing, Networking and Communications (WiMob) (pp. 611-616). IEEE.

Samuel, N., Diskin, T., \& Wiesel, A. (2019). Learning to detect. IEEE Transactions on Signal Processing, 67(10), 2554-2564. IEEE.

Santos Filho, J. C. S., González, D. C., Wolf, A., Mendes, L. L., Yacoub, M. D., \& Fettweis, G. (2018). SNR-Aware Power Allocation Scheme for Lossy-Forward Relaying Systems. 
IEEE Wireless Communications Letters, 7(6), 1018-1021. IEEE.

Sun, H., Chen, X., Shi, Q., Hong, M., Fu, X., \& Sidiropoulos, N. D. (2018). Learning to optimize: Training deep neural networks for interference management. IEEE Transactions on Signal Processing, 66(20), 5438-5453. IEEE.

Thilina, K. M., Choi, K. W., Saquib, N., \& Hossain, E. (2013). Machine learning techniques for cooperative spectrum sensing in cognitive radio networks. IEEE Journal on selected areas in communications, 31(11), 2209-2221. IEEE.

Va, V., Choi, J., Shimizu, T., Bansal, G., \& Heath, R. W. (2017). Inverse multipath fingerprinting for millimeter wave V2I beam alignment. IEEE Transactions on Vehicular Technology, 67(5), 4042-4058. IEEE.

Wang, Y., Narasimha, M., \& Heath, R. W. (2018). MmWave beam prediction with situational awareness: A machine learning approach. 2018 IEEE 19th International Workshop on Signal Processing Advances in Wireless Communications (SPAWC) (pp. 1-5). IEEE.

Wen, C.-K., Shih, W.-T., \& Jin, S. (2018). Deep learning for massive MIMO CSI feedback. IEEE Wireless Communications Letters, 7(5), 748-751. IEEE.
Xiang, W., Johnston, M., \& Le Goff, S. (2017). Low-complexity power control and energy harvesting algorithms for wiretap channels employing finite-alphabet input schemes. IEEE Transactions on Information Forensics and Security, 13(2), 318-326. IEEE.

Xie, S., Zhang, B., Guo, D., \& Zhao, B. (2019). Performance analysis and power allocation for NOMA-based hybrid satellite-terrestrial relay networks with imperfect channel state information. IEEE Access, 7, 136279-136289. IEEE.

Ye, H., Li, G. Y., \& Juang, B.-H. (2017). Power of deep learning for channel estimation and signal detection in OFDM systems. IEEE Wireless Communications Letters, 7(1), 114117. IEEE.

Y $\iota l d \imath$ rım, M., \& Ilhan, H. (2014). Relay selection in OFDM based two-way cooperative systems. 2014 11th International Conference on Electronics, Computer and Computation (ICECCO) (pp. 1-4). IEEE. 